\title{
Viewpoint-invariant Weber fractions and standard contour-curvature discrimination
}

\author{
David H. Foster ${ }^{1}$, Johan Wagemans ${ }^{2}$ \\ ${ }^{1}$ Department of Communication and Neuroscience, Keele University, Staffordshire ST5 5BG, UK \\ 2 Laboratory of Experimental Psychology, University of Leuven, Tiensestraat 102, B-3000 Leuven, Belgium
}

Received: 12 January 1993/Accepted in revised form: 14 May 1993

\begin{abstract}
It is proposed that any cue for the visual discrimination of shape, in particular the discrimination of curved contours, should be such that the perceptual relationships defined by the cue are invariant under changes in observer viewpoint. Such relationships may be quantified by the Weber fraction; that is, the ratio $\Delta c / c$, where, for any particular value $c$ of the cue, $\Delta c$ is the smallest difference in $c$ that can be detected. Eight geometric attributes of curved contours having one symmetry axis and parallel chords (a standard stimulus configuration) were examined for invariance of the Weber fraction under symmetry-preserving affine transformations of the image plane (changes in viewpoint are well approximated by affine transformations when depth is small relative to viewing distance). The attributes, each investigated in previous psychophysical studies, were equivalent-curvature, radius-of-curvature, turning-angle, arc-length-divided-by-chord-length, arc-length, maximumdeviation (sag), area, and mean-deviation. Three of the attributes, namely sag, area, and mean-deviation, satisfied the viewpoint-invariance condition; the remainder failed. These results are considered in relation to previously published empirical data on the Weber fraction for contour-curvature discrimination.
\end{abstract}

\section{Introduction}

The visual system is acutely sensitive to differences in the curvature of edges and of contours in an image (Ogilvie and Daicar 1967; Watt and Andrews 1982; Wilson 1985; Fahle 1986, 1991; Foster et al. 1993), an ability that reflects the importance of contour curvature in determining the perception of two- and three-dimensional physical objects (Attneave 1954; Koenderink and van Doorn 1982; Richards et al. 1986; Lehky and Sejnowski 1988; Link and Zucker 1988). Several experimental analyses have attempted to determine the geometrical attributes of curved lines that might be used in contour-curvature

Correspondence to: D. H. Foster discrimination (e.g. Ogilvie and Daicar 1967; Watt and Andrews 1982; Watt 1984; Wilson 1985; Fahle 1986; Wilson and Richards 1989; Foster et al. 1993; Kramer and Fahle 1993). Attributes considered as candidate cues have included curvature itself, the radius of curvature, the angle turned through by a tangent moving along the curve, the maximum deviation from linearity of the curve (or "sag"), the mean deviation, the area enclosed by the curve, the arc-length of the curve, and the quotient of arclength by chord-length.

Although the question of the identity of the cue for contour-curvature discrimination is ultimately an empirical one, the number of candidate cues can be reduced by the imposition of certain general constraints on the visual comparison of shapes in natural viewing conditions. In Sect. 2, it is argued that, in general, the perceived relationships of shapes, and in particular their discriminability as represented by the Weber fraction, should reflect the corresponding invariances of relationships in an image as the viewpoint of the observer changes in relation to the object or surface. In Sect. 3, changes in observer viewpoint and some viewpoint-invariant properties of surfaces are expressed in terms of the group of affine, symmetry-preserving transformations of the image plane. In Sect. 4, the eight aforementioned candidate cues for contour-curvature discrimination are formally defined, and the effect of each affine transformation on the Weber fraction for each cue is determined. Only three of the candidate cues, namely, sag, area, and mean-deviation, satisfied the viewpoint-invariance condition; the other attributes, including equivalent-curvature, all failed. In Sect. 5, some of these attributes are considered in relation to previously published empirical data on the Weber fraction for contour-curvature discrimination.

\section{Weber's law and viewpoint invariance}

Some motivation for the present approach comes from consideration of visual discrimination in the one-dimensional (achromatic) luminance domain. The discriminability of two lights (self-luminous or reflected) may be summarized by the size of the smallest detectable difference 
$\Delta L=L^{\prime}-L$ between their luminances $L, L^{\prime}$, as a function of $L$, for some criterion level of performance. [The quantity $\Delta L$ is the increment or difference threshold; see Falmagne (1985, p 197); Laming (1986, pp 67-69).] Since the criterion level of performance may be chosen arbitrarily, the increment $\Delta L$ need not be small in relation to $L$. Weber's law (Weber 1834) asserts that the increment $\Delta L$ in $L$ depends linearly on $L$; that is, the Weber fraction $\Delta L / L$ is constant, or, equivalently, $L^{\prime} / L$ is constant. In practice Weber's law holds over a large part of the luminance domain (e.g. Leshowitz et al. 1968). Weber's law is of course not restricted to discriminations of luminance and holds approximately over the greater part of the operating range of many sensory attributes (Laming 1986), and it has been used as a criterion for the acceptability of models of discrimination performance (Falmagne 1985).

Consider a spatially non-uniform surface viewed under a spatially uniform illuminant with illuminance $E$. If $E$ varies (from time to time), the luminance $L$ of the light reflected from the surface also varies, but the relationship between $E$ and $L$ at a given point on the surface remains fixed, determined by the reflection coefficient $r$ (for given directions of incidence and of view at that point); that is, $L=r E / \pi$. Although the luminance $L$ of the surface is not invariant under $E$, the ratio of the luminances from different regions of the surface is. Explicitly, if $L, L^{\prime}$ are the luminances of regions with reflection coefficients $r, r^{\prime}$ respectively, the ratio $L^{\prime} / L$ is $\left(r^{\prime} E / \pi\right) /(r E / \pi)=r^{\prime} / r$, which is a property of the surface, namely the spatial contrast. Therefore, as a visual cue, luminance (the variable $L$ as opposed, for example, to $\log L$ or $L^{2}$ ) has the property that the perceived relationship of two regions, as represented by the Weber fraction for reflected luminance, is invariant under changes in illuminance. Explicitly, $\Delta L / L=\left(L^{\prime}-L\right) / L=\left(r^{\prime} E / \pi-r E / \pi\right) /(r E / \pi)=\left(r^{\prime}-r\right) / r$.

This property - that an invariant perceptual relationship corresponds to an invariant physical ratio - may be applied in the analysis of discriminations in the shape domain. Thus consider how two regions of a surface, or, more specifically, two curved contours on the surface, may be related perceptually in terms of some arbitrary shape attribute. Suppose that from a particular viewpoint the values of this attribute are $c, c^{\prime}$ and that these values are discriminable at some (unspecified) criterion level of performance. As noted earlier, the difference $\Delta c=c^{\prime}-c$ in the values $c, c^{\prime}$ depends on the criterion level of performance, but this level, like other non-geometrical factors, may be assumed to be constant across experimental conditions. The natural changes in the image arise from changes in relative viewpoint of the observer, through changes in position and orientation of the object, or observer, or both. As with luminance, the shape attribute - if it is to provide a cue for shape discrimination rather than a cue just for changes in relative position - should be matched to these natural image changes. More formally, any candidate cue for shape discrimination should satisfy the following invariance condition.

Condition 1. The perceptual relationship of two shapes, expressed by the Weber fraction for the cue, should be invariant under changes in relative viewpoint.
It is assumed that the viewpoint changes are not so extreme that the stimulus becomes too small to be seen or so large that it passes outside the visual field. In some contexts, Condition 1 would be reformulated by saying that the cue should be a relative invariant rather than an absolute invariant, since it involves the ratios of attribute values (Mundy and Zisserman 1992, Sect. 1.1.4). Although Condition 1 is a constraint on candidate cues, it is not sufficient to determine uniqueness, as is shown explicitly later (Sect. 5).

The next section describes how changes in relative viewpoint may be represented as spatial transformations of the image.

\section{Affine transformations and affine invariants}

Assume that the curved contours are drawn on an approximately planar surface, positioned and oriented arbitrarily (but not end-on) in three-dimensional space. Assume also that the surface is sufficiently distant from the eye that the depth of the contours is relatively small so that perspective effects may be ignored (a common assumption in modelling the effects of viewing objects in three-dimensional space, see e.g. Thompson and Mundy 1987; Lamdan et al. 1988; Hopcroft et al. 1992; Wagemans 1993; also Mundy and Zisserman 1992, Sect. 23.10). Changes in viewpoint may then be represented by affine transformations of the fronto-parallel image plane; that is, in the usual Cartesian coordinates, by transformations

$T:(x, y) \rightarrow\left(a_{1} x+b_{1} y+c_{1}, a_{2} x+b_{2} y+c_{2}\right)$,

where, to avoid singularities, the determinant $\operatorname{det}(T)$ $=a_{1} b_{2}-a_{2} b_{1} \neq 0$ (the constants $c_{1}, c_{2}$ should not be confused with the shape-attribute variable $c$ ). In general, under an affine transformation $T$ :

Property 1. Parallel line segments in the plane are mapped onto parallel line segments, and the ratio of their lengths is preserved.

Property 2. Areas of regions in the plane are each scaled by the factor $\operatorname{det}(T)$, and the ratio of any two areas is preserved.

In the previous section, it was proposed (Condition 1) that the Weber fraction $\Delta c / c$ for any candidate cue should be invariant under changes in relative viewpoint. The candidate cues considered here are limited to those which have been investigated in previous psychophysical studies of contour-curvature discrimination (other contour-curvature attributes which have been of theoretical or computational interest, and which exhibit invariance under affine transformations, have been described in e.g. Mundy and Zisserman 1992; Pauwels et al. 1993). Some of the candidate cues can be treated as having either Property 1 or Property 2, and therefore automatically yield Weber fractions that are invariant under affine transformations. For those candidate cues that do not have Property 1 or 2 , the effect of affine transformations must be considered explicitly. 


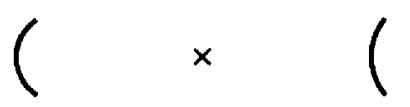

b

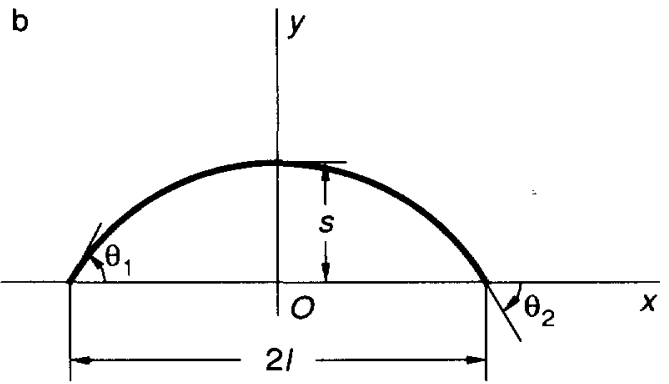

Fig. 1. a Standard stimulus configuration. Only one of the curved lines need be present at a time, and the whole stimulus display may be rotated through $90 \mathrm{deg}$. b Curved-contour parameters: $l$, half chordlength; $s$, height; $\theta_{2}-\theta_{1}$, turning angle. Computational formulas are given in the text (Sect. 4)

Figure 1a abstracts the standard stimulus configurations of previous psychophysical studies (Della Valle et al. 1956; Ogilvie and Daicar 1967; Watt and Andrews 1982; Watt 1984; Wilson 1985; Fahle 1986; Link and Zucker 1988; Wilson and Richards 1989; Foster et al. 1993; Kramer and Fahle 1993), and includes those instances when the curved lines were presented sequentially for comparison, and when there was just one curved line compared with an imaginary straight line. [In the experiments by Wilson (1985), Wilson and Richards (1989), and Link and Zucker (1988) the curved lines had straight-line extensions.] In those studies, the curved lines had one symmetry axis and were positioned with chords parallel to some axis, which, without loss in generality, may be assumed here to be the $x$ - or $y$-axis. Because of this arrangement, it is sufficient to restrict attention to those transformations that are combinations of enlargements (or reductions) $(x, y) \rightarrow(k x, k y)$, with $k>0$, and simple elongations (or compressions) $(x, y) \rightarrow(x, k y)$ or $(x, y) \rightarrow(k x, y)$; that is, transformations $T$ of the form

$T:(x, y) \rightarrow(a x, b y)$,

where the scale factors $a, b>0$. Skew transformations, which do not preserve the symmetry of the curved lines (and which have been investigated in more general studies of form perception; see Wagemans $(1992,1993)$ ), are not included. The transformations of interest are therefore a proper subgroup of the group of affine transformations. (In principle, the spatial separation of the curved lines could also be transformed, but it was either kept fixed or varied randomly in the previously cited experiments.)

The next section considers the effects of these transformations on each of the candidate cues and the ratios of their values.

\section{Cues and transformed cues}

In Fig. 1b, let the arc have the equation $y=f(x)$, $-l \leqslant x \leqslant l$, in the Cartesian coordinate system shown (the endpoints of the arc fall on the $x$-axis). Let the variable height be $s$. For each candidate cue, it is sufficient to evaluate the effect of each transformation $T$ given by (1) on the ratio $c^{\prime} / c$ of any two cue values $c, c^{\prime}\left(c \neq c^{\prime}\right.$, $c \neq 0)$ : if the transformed ratio $T\left(c^{\prime}\right) / T(c)$ is independent of $T$, then, since the Weber fraction at each value $c$ is given by $\left(c^{\prime}-c\right) / c$ for some $c^{\prime}$, the Weber fraction $\left(T\left(c^{\prime}\right)-T(c)\right) / T(c)$ for the transformed curves is also independent of $T$. With an abuse of notation, $T(c)$ is used to represent the value $c$ of the cue after $T$ has been applied to the curve. The arcs are assumed initially to be circular.

\subsection{Equivalent-curvature and radius-of-curvature}

Euclidean curvature has often been used to quantify thresholds in measurements of contour-curvature discrimination (Della Valle et al. 1956; Ogilvie and Daicar 1967; Watt 1984; Wilson 1985), and has been analysed empirically in relation to other cues (Watt and Andrews 1982; Watt 1984; Foster et al. 1993). In general, at each point $(x, f(x))$ on a curve (Fig. 1b), Euclidean curvature (as distinct from affine curvature; Guggenheimer 1977) is defined by

$\frac{\left|f^{\prime \prime}(x)\right|}{\left(1+\left(f^{\prime}(x)\right)^{2}\right)^{3 / 2}}$

where $f^{\prime}(x)$ and $f^{\prime \prime}(x)$ are the first and second derivatives of $f$ at $x$. If the curve is a circular arc, Euclidean curvature (2) is constant along the locus and equal to the reciprocal of the radius of the corresponding circle. Application of each transformation (1), however, yields a non-circular ellipse, the Euclidean curvature of which varies continuously along its locus. One solution is to assign to the transformed curve an "equivalent" curvature, such as the Euclidean curvature of a circular arc with the same chord and sag as the transformed curve (Foster et al. 1993). For curves of 120-deg turning angle (Sect. 4.2), the differences between transformed curves for $a \geqslant 1$ and $b \leqslant 1$ in (1) are small: in the worst case, the maximum deviation in the $y$-direction is not more than $3 \%$ of the height of the best-fitting circular arc (Foster et al. 1993). Thus the cue value $c$ is given by ${ }^{1}$

$c=(1 / l) \sin (2 \arctan (s / l))$.

Under each transformation $T(1)$, this value transforms to

$$
\begin{aligned}
T(c) & =(1 /(a l)) \sin (2 \arctan (b s /(a l))) \\
& =(1 /(a l)) \sin (2 \arctan ((b / a) \tan (\arcsin (l c) / 2))) .
\end{aligned}
$$

\footnotetext{
1 It is also given by $c=2 s /\left(l^{2}+s^{2}\right)$ (the chord theorem), but this formula is less convenient for manipulation
} 
The ratio $c^{\prime} / c$ of two cue values $c, c^{\prime}$ transforms to

$\frac{T\left(c^{\prime}\right)}{T(c)}=\frac{\sin \left(2 \arctan \left((b / a) \tan \left(\arcsin \left(l c^{\prime}\right) / 2\right)\right)\right)}{\sin (2 \arctan ((b / a) \tan (\arcsin (l c) / 2)))}$,

which depends on $a$ and $b$, the scale factors in the $x$ - and $y$-directions respectively (1).

Radius of curvature of a circular arc has also been used to quantify thresholds (e.g. Della Valle et al. 1956; Ogilvie and Daicar 1967). Since it is the reciprocal of equivalent-curvature, the ratio $c^{\prime} / c$ of two cue values $c, c^{\prime}$ for radius-of-curvature is not preserved under affine transformations $T(1)$.

\subsection{Turning-angle}

The angle turned through by a tangent moving along the curve, that is, the turning-angle or "orientation-range", was considered as a possible factor in determining thresholds by Watt (1984), Link and Zucker (1988), Foster et al. (1993), and Kramer and Fahle (1993). It is defined formally by

$\arctan \left(f^{\prime}(l)\right)-\arctan \left(f^{\prime}(-l)\right)$,

that is, $\theta_{2}-\theta_{1}$ in Fig. 1b. The cue value $c$ for a circular arc is given by

$c=4 \arctan (s / l)$.

Under each transformation $T(1)$, this value transforms to

$$
\begin{aligned}
T(c) & =2 \arctan ((b / a) \tan (2 \arctan (s / l))) \\
& =2 \arctan ((b / a) \tan (c / 2)) .
\end{aligned}
$$

The ratio $c^{\prime} / c$ of two cue values $c, c^{\prime}$ transforms to

$\frac{T\left(c^{\prime}\right)}{T(c)}=\frac{\arctan \left((b / a) \tan \left(c^{\prime} / 2\right)\right)}{\arctan ((b / a) \tan (c / 2))}$,

which depends on $a$ and $b$.

\subsection{Arc-length-divided-by-chord-length, and arc-length}

The quotient of arc-length by chord-length and arclength alone were each considered as candidate cues by Foster et al. (1993). Arc-length-divided-by-chord-length is defined by

$\frac{1}{2 l} \int_{-l}^{l}\left(1+\left(f^{\prime}(x)\right)^{2}\right)^{1 / 2} \mathrm{~d} x$

The cue value $c$ for a circular arc is given by

$c=\frac{2 \arctan (s / l)}{\sin (2 \arctan (s / l))}$.

Under each transformation $T(1)$, this value transforms to

$T(c)=\frac{E\left(2 \arctan (s / l), 1-b^{2} / a^{2}\right)}{\sin (2 \arctan (s / l))}$,

where $E(\phi, m)$ is an elliptic integral of the second kind, $E(\phi, m)=\int_{0}^{\phi}\left(1-m \sin ^{2} \theta\right)^{1 / 2} \mathrm{~d} \theta$, and $s$ is given implicitly by (5). The ratio $c^{\prime} / c$ of two cue values $c, c^{\prime}$ transforms to

$$
\frac{T\left(c^{\prime}\right)}{T(c)}=\frac{\sin (2 \arctan (s / l)) E\left(2 \arctan \left(s^{\prime} / l\right), 1-b^{2} / a^{2}\right)}{\sin \left(2 \arctan \left(s^{\prime} / l\right)\right) E\left(2 \arctan (s / l), 1-b^{2} / a^{2}\right)},
$$

where $s, s^{\prime}$ are given by (5) for $c=c, c^{\prime}$ respectively. The ratio $T\left(c^{\prime}\right) / T(c)$ depends on $a$ and $b$. The same transformed ratio (6) is obtained for arc-length alone.

\subsection{Sag}

Threshold measurements based on the perpendicular distance from the midpoint of the curve to the chord joining its ends were made by Della Valle et al. (1956), Ogilvie and Daicar (1967), Andrews et al. (1973), Watt and Andrews (1982), Foster (1983), Ferraro and Foster (1986), Fahle (1986, 1991), and Foster et al. (1993). This attribute has been given several names: "sagitta" ${ }^{2}$ (Della Valle et al. 1956) (and commonly "sag"); "height", for a horizontal curved line (Watt and Andrews 1982); and "arcchord distance" (Phillips and Rosenfeld 1987). In Fig. 1b, the sag of the curve is $s$, and the cue value $c$ is defined by $c=s$.

Since the curves under comparison have the same orientation in the plane, by Property 1 of Sect. 3 the ratio $c^{\prime} / c$ of two cue values $c, c^{\prime}$ is invariant under each transformation $T$ (1). Explicitly,

$\frac{T\left(c^{\prime}\right)}{T(c)}=\frac{c^{\prime}}{c}$

which is independent of $a$ and $b$.

\subsection{Area and mean-deviation}

The area enclosed by the curve and the chord joining its ends was suggested by Ogilvie and Daicar (1967) as an appropriate measure for curvature acuity. It was also considered by Foster et al. (1993). With respect to Fig. 1b, it is defined by

$\int_{-l}^{l} f(x) \mathrm{d} x$.

The cue value $c$ for a circular arc is given by

$c=\frac{l^{2}(4 \arctan (s / l)-\sin (4 \arctan (s / l)))}{2 \sin ^{2}(2 \arctan (s / l))}$.

By Property 2 of Sect. 3, the ratio $c^{\prime} / c$ of two cue values $c, c^{\prime}$ is invariant under each transformation $T$ (1). Explicitly,

$$
\begin{aligned}
& \frac{T\left(c^{\prime}\right)}{T(c)} \\
& =\frac{\sin ^{2}(2 \arctan (s / l))\left(4 \arctan \left(s^{\prime} / l\right)-\sin \left(4 \arctan \left(s^{\prime} / l\right)\right)\right)}{\sin ^{2}\left(2 \arctan \left(s^{\prime} / l\right)\right)(4 \arctan (s / l)-\sin (4 \arctan (s / l)))},
\end{aligned}
$$

\footnotetext{
${ }^{2}$ From the Latin for arrow
} 
where $s, s^{\prime}$ are given implicitly by (8) for $c=c, c^{\prime}$ respectively. The ratio $T\left(c^{\prime}\right) / T(c)$ is independent of $a$ and $b$.

The mean-deviation of the curve is the quotient of area by chord-length, and is defined by

$\frac{1}{2 l} \int_{-l}^{l} f(x) \mathrm{d} x$.

The same transformed ratio (9) is obtained for meandeviation.

\section{Discussion}

Since none of the transformed Weber fractions contained either of the transformation scale factors $a, b$ of (1) except as a ratio $b / a$, all of the curved-line attributes yielded Weber fractions (3), (4), (6), (7), (9) that were invariant under enlargements and reductions, $(x, y) \rightarrow(k x, k y)$, of the image. Five of the attributes yielded Weber fractions that were not invariant under combinations of enlargements or reductions and simple elongations or compressions: $(x, y) \rightarrow(x, k y)$ and $(x, y) \rightarrow(k x, y)$; these attributes were equivalent-curvature (3), radius-of-curvature, turning-angle (4), arc-length-divided-by-chord-length (6), and arc-length. Sag (7), area (9), and mean-deviation were all invariant under elongations and compressions.

How consistent with empirical data on contour-curvature discrimination is this set of cues? Several previous studies have established critical limits on discrimination performance, but have not determined unambiguously the identity of the cue (or possible cues) for contourcurvature discrimination (Ogilvie and Daicar 1967; Watt and Andrews 1982; Watt 1984; Wilson 1985; Koenderink and Richards 1988; Wilson and Richards 1989; Kramer and Fahle 1993).

One information-theoretically oriented approach to this problem has been to determine for each geometric attribute of interest a "relative efficiency" with respect to an ideal observer. This observer was assumed to be subject to several implicit errors, of which one of the most important was the error in its knowledge of each light receptor's position; these errors were assumed to be normally distributed and independent of each other (Andrews et al. 1973). The ideal observer was, however, assumed to know that the stimulus was a circular arc. The task was then simply to determine the value of the selected attribute that gave the best-fitting arc to the data points sampled by the retina (Watt and Andrews 1982). It was found that under some conditions increasing curvature led to increasing relative efficiency, and it was suggested (Watt 1984) that the mechanism involved in discriminating curves operates by calculating curvature; other attributes, including turning-angle, sag, and chordlength, were all rejected.

Another approach, more operationally oriented, has been to consider the problem as one of statistical estimation theory (Fisher 1992; Stuart and Ord 1991); that is, to determine which of a set of curved-line attributes gave the best estimate of increment threshold $\Delta c$ (and of the standard deviation of increment threshold) as a function of cue value $c$ (Foster et al. 1993). The best estimator in the sense of statistical estimation theory is the most efficient one, that is, the one associated with the smallest variance in the data. Notice that this notion of efficiency concerns the predictability of the data, whereas the relative efficiency notion used by Watt and Andrews (1982) concerns the relationship of the data to those obtainable by a particular detector.

In a series of experiments (Foster et al. 1993), seven of the eight geometric attributes considered here were assessed: equivalent-curvature, turning-angle, arc-length, arc-length-divided-by-chord-length, sag, area, and meandeviation. The experiments each required the discrimination of two simultaneously presented, 1-s-duration, curved-line stimuli with parallel chords, whose chordlengths ranged from 12 arcmin to 48 arcmin visual angle and whose curvatures ranged from $0 \operatorname{arcmin}^{-1}$ to 0.13 $\operatorname{arcmin}^{-1}$. For each candidate cue, the increment threshold $\Delta c$ was determined as a function of $c$ under combinations of enlargements or reductions and simple elongations or compressions of the curved lines. Figure 2 shows a set of data from one of the experiments (Foster et al. 1993, Fig. 2), with corresponding data - previously unpublished - for radius-of-curvature (for which infinite values, at zero equivalent-curvature, have been suppressed). Estimates of $\Delta c$ are plotted as a function of $c$ for the eight curved-line attributes. The different symbols correspond to different enlargements of the curved lines, as indicated in the legend to the figure. For each of the curved-line attributes, the data were subjected to an analysis of variance with repeated measures and an analysis of linear and higher-order (up to quartic) trends with respect to $c$ (Winer 1971). The broken lines in Fig. 2 show only linear least-squares regressions.

For this data set and others (Foster et al. 1993), sag and its approximation, mean-deviation, accounted well for the variance in the data; sag was the best predictor, and its increment-threshold function satisfied Weber's law (Fig. 2f) over almost all of the stimulus range. Area, despite its theoretical admissibility in the present context and its empirical acceptability for curved-line enlargements (Fig. 2g), failed significantly to account for the variance in some other experimental conditions (Foster et al. 1993, Figs $3 \mathrm{~g}, 4 \mathrm{~g}$ of that study). Notice, however, that unlike area, sag fails to yield a relative invariant when the curved lines do not have parallel chords (Property 1 , Sect. 3); that is, in the non-standard stimulus arrangement, the ratio of two sag values is not constant under affine transformation. A further experimental test might thus entail determining discrimination thresholds for pairs of curved lines with chords at different relative orientations: if sag is indeed the preferred cue, then the variance of the thresholds should increase in the nonparallel condition. Some data consistent with this prediction have been reported in experiments on curved-line texture segmentation, but using short display durations (Simmons and Foster 1992).

Display duration is an important factor in influencing the form of the dependence of $\Delta c$ on $c$. With shortduration (100-ms) displays, followed by a random-dot mask, discrimination performance has been found to 

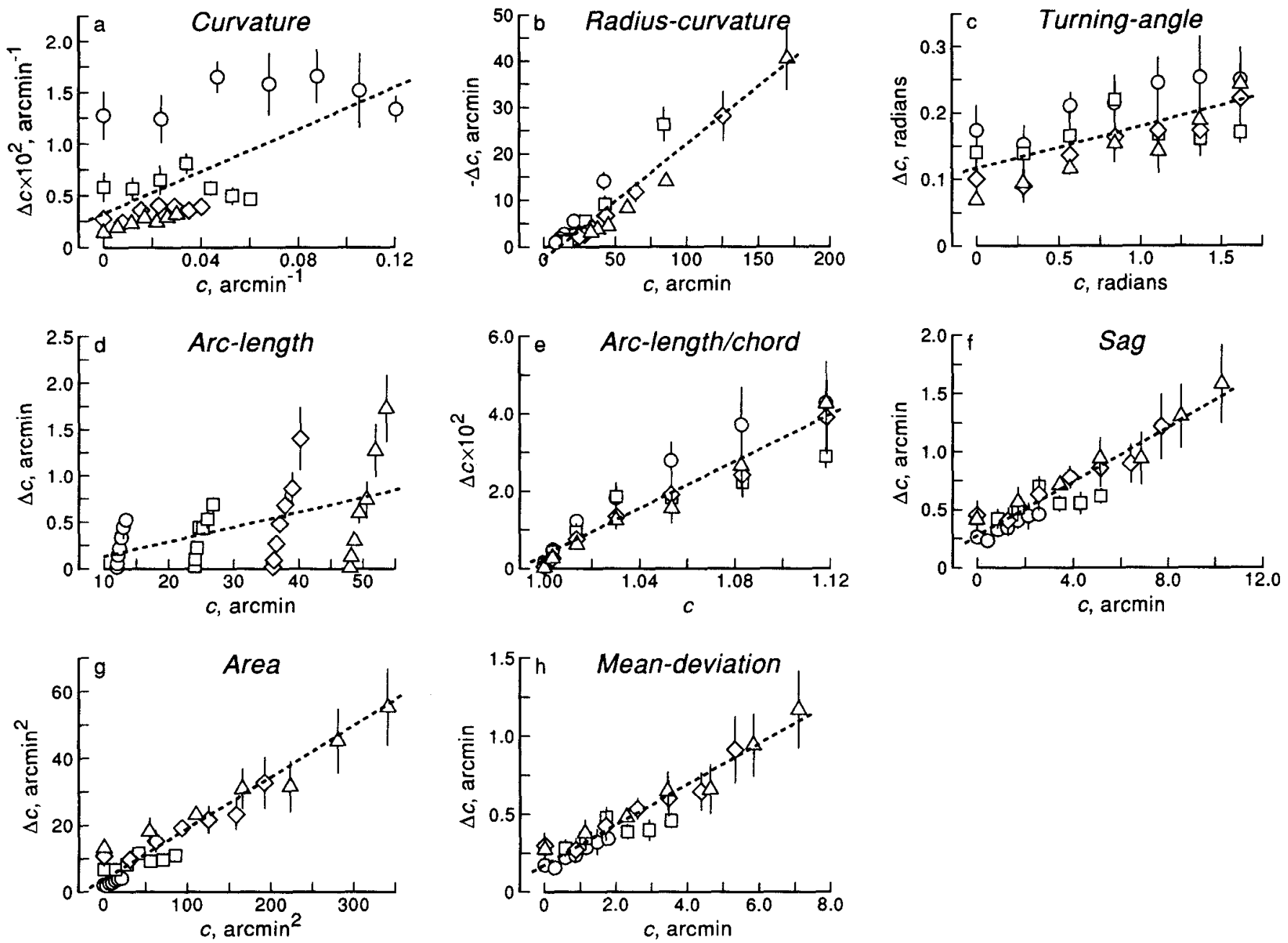

Fig. 2a-h. Increment-threshold functions for curved-line discrimination [adapted from Fig. 2, Foster et al. (1993), with previously unpublished data for radius of curvature]. For each of the specified curved-line attributes, estimates of increment threshold $\Delta c$ are plotted against reference value $c$ for four chord lengths: $0,0.2 \mathrm{deg} ; \square, 0.4 \mathrm{deg} ; \diamond, 0.6 \mathrm{deg}$; and $\triangle, 0.8 \mathrm{deg}$. Each data point is the weighted mean over five subjects, and the vertical bars show \pm 1 SEM. Broken lines are least-squares linear regressions. Sag, mean-deviation and area $(\mathbf{f}-\mathbf{h})$ all accounted well for the variance in the data $[F(26,108) \leqslant 1.04]$; curvature and arc-length $(\mathbf{a}, \mathbf{d})$ both failed highly significantly $[F(26,108) \geqslant 7.06]$, as did radius-of-curvature (b) despite the suppression of infinite values $[F(22,92)=2.57]$. When other experimental conditions were included, turning-angle (c) and arc-length-divided-bychord-length (e) also failed highly significantly $[F(26,81) \geqslant 2.95]$, and only sag and mean-deviation provided acceptable fits overall (Foster et al. 1993)

vary rapidly and non-monotonically with sag. In one study (Foster 1983), two peaks were obtained, the one at sag 0.87 arcmin and the other at sag 1.81 arcmin, and were considered to relate to two (possibly three) discrete mechanisms for curved-line coding. As stimulus duration was increased (from $60 \mathrm{~ms}$ to $2 \mathrm{~s}$ ), discrimination performance was found to become progressively flatter; and at stimulus durations of 1-2 $\mathrm{s}$ linear performance was obtained (Ferraro and Foster 1986; Foster and Cook 1989). A two-layer neuronal model of the underlying processes was proposed by Costa and Ferraro (1993). It successfully accounted for discrete and continuous modes of curved-line discrimination performance as display duration was varied.

In applications where curved lines may extend to the limits of the visual field, the domain of definition of sag, mean-deviation, and area must be constrained. For curved contours of non-constant curvature, points of inflexion and local curvature extrema may be used computationally to define the effective extent of a contour segment (but notice that local curvature extrema are not themselves affine invariant). Sag has been used in machine-vision applications to partition and approximate curves. Thus, points may be selected at which the distance of the curve from one or more chords has a local maximum (e.g. Phillips and Rosenfeld 1987); or the curve may be approximated by a polygon with a given number of vertices such that the maximum distance of the curve from the polygon satisfies some goodness-of-fit criterion (e.g. Ramer 1972). In the design of psychophysical experiments, sag has been used to parameterize transformationally uniform families of stimuli; that is, families of stimuli in which equal increments in the parameter correspond to stimulus changes that in some sense are equal (Foster 1980). Formally, transformational uniformity is achieved by requiring that the family of stimuli should be 
generated by a local one-parameter group of local transformations. Transformationally uniform scales have been exploited in studies of pattern discrimination (e.g. Cermak 1977; Foster 1980, 1983; Ferraro and Foster 1986); pattern matching in two or three dimensions (Wagemans 1993); and visual apparent motion (Foster 1975, 1978; Farrell and Shepard 1981).

The present analysis identifying sag, mean-deviation, and area as attributes that yield invariant contour-curvature discrimination does not of course imply that performance must always be determined by one of these cues. Clearly, if curves have the same sag values and sufficiently different chord-lengths, or arc-lengths, they may still be discriminated. Nor does this analysis imply that there are no other attributes that might be used for invariant contour-curvature discrimination. There are trivial combinations of, for example, sag and mean-deviation that yield viewpoint-invariant Weber fractions. More fundamentally, there are several attributes of curved contours involving first- and higher-order derivatives that give absolute or relative invariants under affine or projective transformations (Weiss 1992; Moons et al. 1993; Pauwels et al. 1993). These differential and semidifferential invariants automatically satisfy Condition 1. For their candidacy as cues for contour-curvature discrimination to be evaluated further, some other measure would need to be introduced, for example, the statistical efficiencies of the attributes in relation to empirical increment-threshold functions, as was done in Foster et al. (1993) for the attributes considered here.

Acknowledgements. We are grateful to P.C. Dodwell, J.J. Koenderink, D.R.J. Laming, D.R. Simmons, G. Sparr, and A. Zisserman for advice and to P.J. Giblin, T. Moons, E.J. Pauwels, G. Sparr, M.G.A. Thomson, L.J. Van Gool, and A. Zisserman for critical reading of the manuscript. This work was supported by EC Esprit Basic Research Action No. 6448 (VIVA). J.W. was supported by the National Fund for Scientific Research (Belgium).

\section{References}

Andrews DP, Butcher AK, Buckley BR (1973) Acuities for spatial arrangement in line figures: human and ideal observers compared. Vision Res 13:599-620

Attneave F (1954) Some informational aspects of visual perception. Psychol Rev 61:183-193

Cermak GW (1977) Performance in a delayed comparison discrimination task as a function of stimulus interpretation. Percept Psychophys $21: 69-76$

Costa T, Ferraro M (1993) A neural model of discrete and continuous modes of visual discrimination. Spatial Vision 7:243-255

Della Valle L, Andrews TG, Ross S (1956) Perceptual thresholds of curvilinearity and angularity as functions of line length. J. Exp Psychol 51:343-347

Fahle M (1986) Curvature detection in the visual field and a possible physiological correlate. Exp Brain Res 63:113-124

Fahle M (1991) Parallel perception of vernier offsets, curvature, and chevrons in humans. Vision Res 31:2149-2184

Falmagne J-C (1985) Elements of psychophysical theory. Clarendon Press, Oxford

Farrell JE, Shepard RN (1981) Shape, orientation, and apparent rotational motion. J Exp Psychol [Hum Percept] 7:477-486

Ferraro M, Foster DH (1986) Discrete and continuous modes of curved-line discrimination controlled by effective stimulus duration. Spatial Vision 1:219-230
Fisher RA (1922) On the mathematical foundations of theoretical statistics. Philos Trans R Soc London Ser A 222:309-368

Foster DH (1975) Visual apparent motion and some preferred paths in the rotation group $\mathrm{SO}(3)$. Biol Cybern 18:81-89

Foster DH (1978) Visual apparent motion and the calculus of variations. In: Leeuwenberg ELJ, Buffart HFJM (eds) Formal theories of visual perception. Wiley, Chichester, pp 67-82

Foster DH (1980) A spatial perturbation technique for the investigation of discrete internal representations of visual patterns. Biol Cybern 38:159-169

Foster DH (1983) Visual discrimination, categorical identification, and categorical rating in brief displays of curved lines: implications for discrete encoding processes. J Exp Psychol [Hum Percept] 9:785-806

Foster DH, Cook MJ (1989) Categorical and noncategorical discrimination of curved lines depends on stimulus duration, not performance level. Perception 18:519

Foster DH, Simmons DR, Cook MJ (1993) The cue for contour-curvature discrimination. Vision Res 33:329-341

Guggenheimer HW (1977) Differential geometry. Dover, New York (original publication McGraw-Hill, New York, 1963)

Hopcroft JE, Huttenlocher DP, Wayner PC (1992) Affine invariants for model-based recognition. In: Mundy JL, Zisserman A (eds) Geometric invariance in computer vision. MIT Press, Cambridge, Mass, pp 354-374

Koenderink JJ, Doorn AJ van (1982) The shape of smooth objects and the way contours end. Perception 11:129-137

Koenderink JJ, Richards W (1988) Two-dimensional curvature operators. J Opt Soc Am [A] 5:1136-1141

Kramer D, Fahle M (1993) On curvature detection in lines and edges. Invest Ophthalmol Vis Sci 34:778

Lamdan Y, Schwartz JT, Wolfson HJ (1988) Object recognition by affine invariant matching. In: Proceedings of Computer Society Conference on Computer Vision and Pattern Recognition, Ann Arbor, MI, IEEE, pp 335-344

Laming D (1986) Sensory analysis. Academic Press, London

Lehky SR, Sejnowski TJ (1988) Network model of shape-from-shading: neural function arises from both receptive and projective fields. Nature 333:452-454

Leshowitz B, Taub HB, Raab DH (1968) Visual detection of signals in the presence of continuous and pulsed backgrounds. Percept Psychophys 4:207-213

Link NK, Zucker SW (1988) Corner detection in curvilinear dot grouping. Biol Cybern 59:247-256

Moons T, Pauwels EJ, Van Gool LJ, Oosterlinck A (1993) Foundations of semi-differential invariance and its application to recognition under affine distortion. Int $\mathbf{J}$ Comput Vision (in press)

Mundy JL, Zisserman A (eds) (1992) Geometric invariance in computer vision. MIT Press, Cambridge, Mass

Ogilvie J, Daicar E (1967) The perception of curvature. Can J Psychol $21: 521-525$

Pauwels EJ, Moons T, Van Gool LJ, Kempenaers P, Oosterlinck A (1993) Recognition of planar shapes under affine distortion. Int J Comput Vision (in press)

Phillips T-Y, Rosenfeld A (1987) A method of curve partitioning using arc-chord distance. Pattern Recognition Lett 5:285-288

Ramer U (1972) An iterative procedure for the polygonal approximation of plane curves. Comput Graphics Image Processing 1:244-256

Richards W, Dawson B, Whittington D (1986) Encoding contour shape by curvature extrema. J Opt Soc Am [A] 3:1483-1491

Simmons DR, Foster DH (1992) Segmenting textures of curved-line elements. In: Orban GA, Nagel H-H (eds) Artificial and biological vision systems. Springer, Berlin Heidelberg New York, pp 324-349

Stuart A, Ord JK (1991) Kendall's advanced theory of statistics. (Classical inference and relationship, vol 2, 5th edn). Edward Arnold, London

Thompson DW, Mundy JL (1987) 3-Dimensional model-matching from an unconstrained viewpoint. In: Proc IEEE Int Conf on Robotics and Automation, Raleigh, NC, IEEE, pp 208-220

Wagemans J (1992) Perceptual use of nonaccidental properties. Can J Psychol 46:236-279

Wagemans J (1993) Skewed symmetry: a nonaccidental property used to perceive visual forms. J Exp Psychol [Hum Percept] $19: 364-380$ 
Watt RJ (1984) Further evidence concerning the analysis of curvature in human foveal vision. Vision Res 24:251-253

Watt RJ, Andrews DP (1982) Contour curvature analysis: hyperacuities in the discrimination of detailed shape. Vision Res 22:449-460

Weber EH (1834) De Tactu. Koehler, Leipzig

Weiss I (1992) Noise resistant invariants of curves. In: Mundy JL, Zisserman A (eds) Geometric invariance in computer vision. MIT Press, Cambridge, Mass, pp 135-156
Wilson HR (1985) Discrimination of contour curvature: data and theory. J Opt Soc Am [A] 2:1191-1198

Wilson HR, Richards WA (1989) Mechanisms of contour curvature discrimination. J Opt Soc Am [A] 6:106-115

Winer BJ (1971) Statistical principles in experimental design, 2nd edn. McGraw-Hill, New York 